\title{
A prospective study of a cohort of patients with necrotizing fasciitis at a level 2 hospital in Sri Lanka
}

\author{
Janaka Perera, Piyal Kurukulasooriya \\ Surgical Department, District General Hospital Polonnaruwa
}

Keywords: Necrotizing fasciitis; atypical presentations

\begin{abstract}
Necrotizing fasciitis is a rapidly progressive inflammation and necrosis of soft tissues and fascia, accompanied by severe systemic toxicity. In some cases early diagnosis is challenging. Objective of this study is to identify basic investigations that will aid in early diagnosis in such equivocal cases.
\end{abstract}

This is a prospective study. Patients who underwent wound debridement for clinically diagnosed necrotizing fasciitis and suspected necrotizing fasciitis at a level 2 hospital in Sri Lanka were included in the study. All together there were 24 patients who were diagnosed to have necrotizing fasciitis with intraoperative findings. In all patients we did full blood count [FBC], C-reactive protein [CRP], random blood sugar [RBS], Arterial blood gas analysis [ABG], plain x-ray films of affected area and wound swab culture and ABST before surgical intervention. Mean age was 67.2 [range: 38-85] years. Diabetes mellitus was a co-morbidity in $67 \%$ of patients. Commonest affected area was the lower limbs. Out of all patients $62 \%$ of patients had a history of muddy water contact. All the patients had WBC more than 15,000 where as $58 \%$ of patients had WBC more than 25,000 . CRP was more than 150 in $83 \%$ patients. Among all $54 \%$ of patients' plain $\mathrm{x}-$ rays were positive for soft tissue gas. Majority of wound swab cultures were poly microbial. Commonest organisms were Streptococcus species, Staphylococcus and enterobacteriaceae.

Overall death rate was $42 \%$ in this study. Among 14 patients who were diagnosed on admission, 3 patients died. Death rate was $21 \%$. Among 10 patients whose diagnoses got delayed, 7 patients died. Death rate was $70 \%$.

A high clinical suspicion of necrotizing fasciitis is needed in an elderly patient presenting with cutaneous infection causing swelling, pain and erythema, with co-morbidity of diabetes.

Correspondence: Janaka Perera

E-mail: asankajanaka@ymail.com

(iDhttps://orcid.org/0000-0002-0764-9959

Received: 20-12-2020 Accepted: 30-03-2021

DOI: http://doi.org/10.4038/sljs.v39i1.8783
High WBC, High CRP and presence of soft tissue gas in plain $\mathrm{x}$-rays are highly suggestive for underline necrotizing fasciitis. Diagnostic score for Necrotising fasciitis can be made using these basic investigations and $\mathrm{x}$-ray findings which need to be validated in further studies.

\section{Introduction}

Necrotizing fasciitis is a rapidly progressive inflammation and necrosis of fascia, underlying muscle, subcutaneous fat and overlying skin which is often accompanied by severe systemic toxicity[1]. Infection typically starts and spreads along the muscle fascia due to its relatively poor blood supply; muscle tissue is frequently spared because of its generous blood supply. Initially, the overlying tissue can appear unaffected; therefore, necrotizing fasciitis is difficult to diagnose without direct visualization of the fascia. It is first described by Hippocrates in the fifth century B.C. Although the incidences of necrotizing fasciitis are ranging between 0.3 to 15 per 100,000 population all over the world, mortality rates are very high. Irrespective of the advances of care case fatality rate is approximately $30 \%$ and over many years it has remained almost unchanged[1].

Necrotizing infection most commonly involves the extremities [lower extremity more commonly than upper extremity][2]. Other presentations are necrotizing fasciitis of the perineum [Fournier's gangrene], head and neck region. Usually there is preceding event like major penetrating trauma, minor laceration or recent surgery.

Necrotizing infection usually presents acutely [over hours]; rarely, it may present sub acutely [over days]. Rapid progression can occur, leading to systemic toxicity, limb loss, and death.[1] Common clinical manifestations include erythema, oedema, severe pain, fever, crepitus, skin bullae, necrosis, or ecchymosis.Conditions which can presents in the same way are cellulitis, deep vein thrombosis etc.

Laboratory findings are generally nonspecific. Abnormalities may include leucocytosis, acidosis, coagulopathy, hyponatremia, elevated inflammatory markers [C-reactive protein and/or erythrocyte sedimentation rate], and elevations in serum creatinine, lactate, creatine kinase [CK], and aspartate aminotransferase [AST][1]. Risk Indicator for 
Necrotizing Fasciitis [LRINEC] score has been described; it is based on laboratory indicators including white cell count, haemoglobin, sodium, glucose, creatinine, and C-reactive protein. The tool was initially noted to have high specificity and negative predictive value; however, subsequent studies have demonstrated limited sensitivity[3].

Although classically a clinical diagnosis, imaging is a powerful adjunct to facilitate early diagnosis in equivocal cases. The findings of dissecting gas along fascial planes in the absence of trauma in plain x-rays is a specific sign, but is only seen in $24.8-55.0 \%$ of patients[2]. Compared to plain radiography, $\mathrm{CT}$ provides higher sensitivity [80\%]. But compared to CT, plain x-rays are freely available and easily accessible in level 2 hospitals here.

The diagnosis of necrotizing infection is established via surgical exploration of the soft tissues in the operating room[1]. Intraoperative specimens should be sent for Gram stain and culture. Multiple bacteria are often involved. Based on the etiology it is divided into two main types which are polymicrobial and monomicrobial. In polymicrobial type anaerobic species [most commonly Bacteroides, Clostridium, or Peptostreptococcus] is isolated in combination with Enterobacteriaceae [Escherichia coli, Enterobacter, Klebsiella, Proteus etc] and one or more facultative anaerobic streptococci [other than group A Streptococcus [GAS]]. In monomicrobial type necrotizing infection is usually caused by GAS or other beta-hemolytic streptococci[1].

Diabetes, Immunosuppression, advanced age, Chronic systemic diseases [renal failure, atherosclerosis, and hypertension] are considered as predisposing factors [5].

Necrotizing fasciitis is a surgical emergency. The degree of suspicion should be high since the clinical presentation is variable and prompt intervention is critical. The lay press has referred to organisms that cause NSTI as "flesh-eating bacteria"[1].Treatment includes early diagnosis, prompt surgical debridement and parenteral antibiotics[6].

In some atypical cases early diagnosis is challenging and result a delay in diagnosis and treatment. Basic laboratory investigations [FBC, CRP, RBS, and Serum Lactate] might helpful in diagnosis of atypical presentations. Although not routinely performed presence of soft tissue gas and inflammatory changes in plain x-ray might help in early diagnosis of such atypical presentations in resource limited settings. To correlate the possibilities if there are any, between those freely available and cost effective investigations which are available even in a resource poor setting towards early diagnosis of necrotizing fasciitis is the purpose of this study.

\section{Objectives}

Objectives of this study are to correlate basic laboratory investigations, importance of positive plain x-ray findings to formulate a combined diagnostic score with basic laboratory tests and plain $\mathrm{x}$-rays towards early diagnosis of atypical presentations and to identify common causative organisms for necrotizing fasciitis.

\section{Methodology}

This was a prospective study. Patients who underwent wound debridement for clinically diagnosed necrotizing fasciitis in the extremities and suspected necrotizing fasciitis in the extremities at a level 2 hospital in Sri Lanka from August 2019 to March 2020 were included in the study. In all patients we did full blood count [FBC], C-reactive protein [CRP], random blood sugar [RBS], arterial blood gas analysis [ABG], plain x-ray films of affected area and wound swab culture and ABST before surgical intervention. Laboratory data, x-ray findings, comorbidities and outcomes were collected for each patient and extracted for analysis.

\section{Results}

A total of 26 patients who were clinically diagnosed to have necrotizing fasciitis underwent surgical intervention. Out of that 24 patients were diagnosed to have necrotizing fasciitis by intraoperative findings of soft tissue necrosis and infection. Only those with confirmed intraoperative diagnosis were included in the study. Among those diagnosed patients 14 patients were diagnosed on admission by clinical presentation while rests of 10 patients' diagnoses were delayed until septic features were developed because of their atypical initial presentations.

There was no delay in treatments for clinically apparent necrotizing fasciitis whose first surgical debridement were done in the same day in few hours of admission. Delay in offering initial debridement for certain cases were only because delay in diagnosis due to atypical presentations. Subsequent wound debridements were done without any delay depending on patients' clinical condition and wound status.

Out of them 17 were males and 7 were females. Mean age was 67.2 [range: 38-85] years. Diabetes mellitus was a co-morbidity in $67 \%$ of patients. Commonest affected site was the lower limbs which was 21 out of 24 . Other 3 patients had disease involvement in their upper limbs. Among those 21 patients 15 patents had minor skin breach. None of patients had major blunt or penetrating trauma or any surgical procedures in that affected limb in the recent past. Among those 21 patients $62 \%$ had history of muddy water contact. 


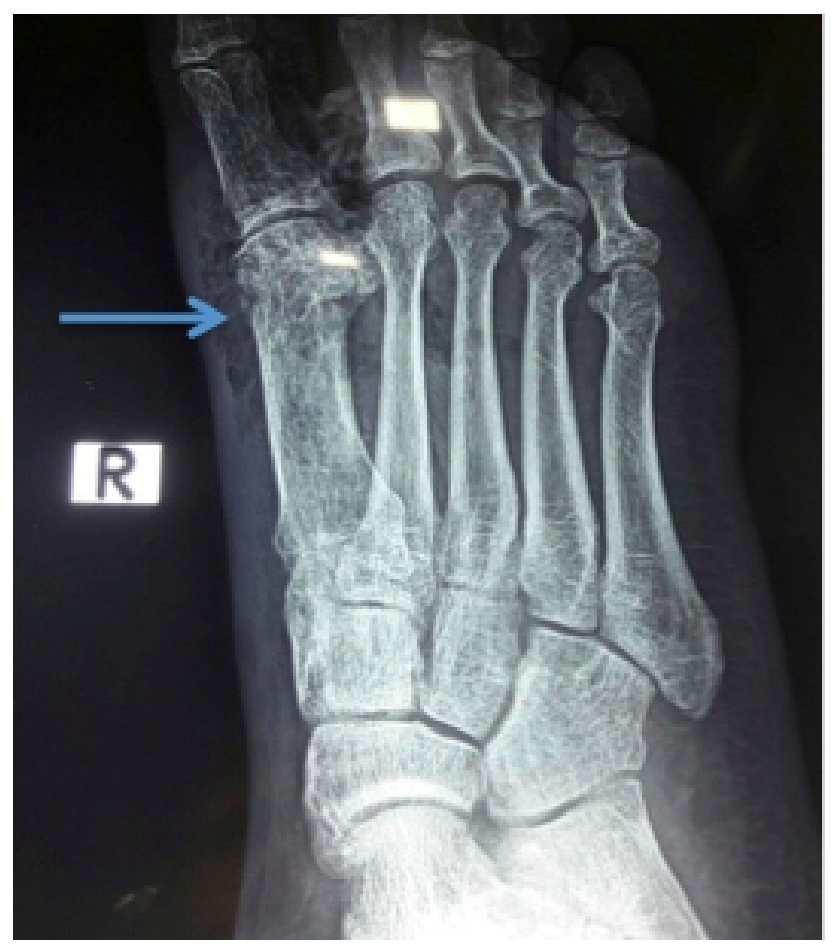

Figure 1. AP view of right foot showing soft tissue gas [indicated by arrow]

All the patients had WBC more than 15,000 where as 58\% of patients had WBC more than 25,000. CRP was more than 150 in $83 \%$ patients.

Among 14 patients who were clinically diagnosed to have necrotizing fasciitis on admission 7 patients had soft tissue gas in their plain x-rays. Among patients whose diagnosis got delayed 6 out of 10 patients had soft tissue gas in their plain $\mathrm{x}-$ ray films. Among all, 54\% of patients' plain x-ray films were positive for soft tissue gas [Figure 1].

Mortality in our study was $42 \%$ [n=10]. Among 14 patients who were diagnosed on admission, 3 patients died. Among 10 patients whose diagnoses got delayed, 7 patients died.

Majority of wound swab cultures were poly microbial. Commonest organisms identified were Streptococcus species, Staphylococcus aureus, Enterobacteriaceae, Pseudomonas aeruginosa and Clostridium species.

\section{Discussion}

Necrotizing fasciitis is a rare but very destructive invasive infection of skin, subcutaneous tissues and deep fascia[1]. It is often polymicrobial[7]. Diabetes, Immunosuppression, advanced age, Chronic systemic diseases [renal failure, atherosclerosis, and hypertension] are considered as predisposing factors[1]. Early diagnosis is lifesaving in managing necrotizing fasciitis patients. Treatment includes prompt surgical debridement, parenteral antibiotics and nutritional support.
Results of our study

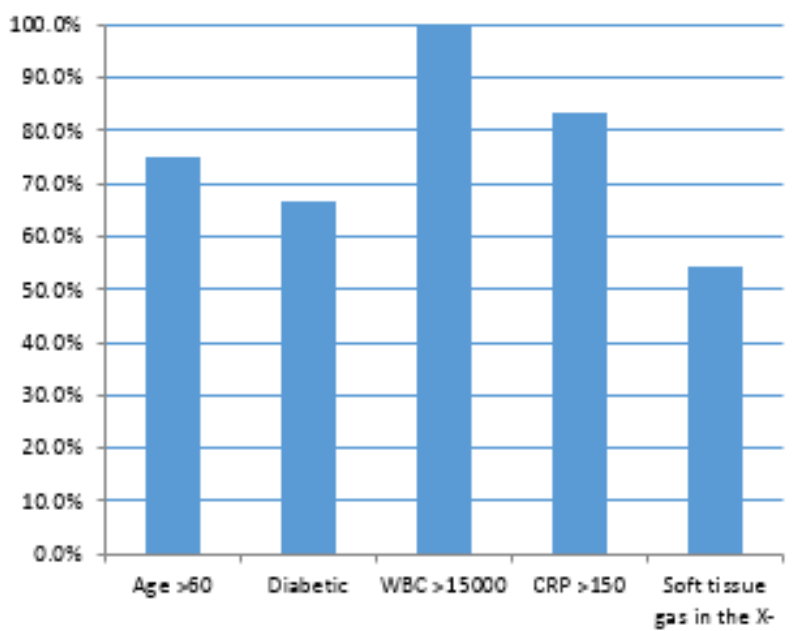

ray

It appears from the data in this study, the disease is commoner in males than females with the mean age 67.2 years. Similar studies also reported that the majority of patients were male and the mean age was between 50 to 60 years in those studies[8]. In this study most commonly affected site was the lower extremities probably due to most of patients in this agricultural area were farmers with a history of muddy water contact. A systemic review of similar studies which has done previously also shows lower extremity is commoner than the upper extremity in regard necrotizing fasciitis[2].

Out of 24 patients 14 patients had necrotizing fasciitis which we could diagnosed clinically on admission. 10 patients had necrotizing fasciitis which were difficult to differentiate from cellulitis like soft tissue infection whose clinical diagnoses were delayed until features of sepsis develop.

Case fatality rate reported in the literature varies widely. A systemic review shows it's up to 34\%[8]. Mortality rate in our study was $42 \%$ [n=10. Among 14 patients who were diagnosed on admission, 3 patients died. Death rate was $21 \%$. Among 10 patients whose diagnoses got delayed, 7 patients died. Death rate was $70 \%$. Mortality rate in patients who were diagnosed on admission is significantly low compared to the patients whose diagnosis was delayed $[\mathrm{p}<0.01]$.

Plain x-rays are not routinely used with a diagnostic intention in necrotising fasciitis. But presence of soft tissue gas in a radiograph with clinical features suggestive of soft tissue infection is highly suggestive for necrotizing fasciitis[9]. Although CT is much more sensitive than plain radiographs in identifying soft tissue gas, it is not freely available, especially in a level 2 hospital in Sri Lanka. Conversely plain radiographs are freely available, less time consuming and result in less radiation exposure. In several previous studies with similar interest we identified presence of soft tissue gas 
in plain x-rays were ranging from $20 \%$ to $50 \%$ [5]. Percentage of patients in our study who had soft tissue gas in the plain $\mathrm{x}$ rays [54\%] was similar to the previous studies. Out of 10 patients whose diagnoses were delayed due to atypical presentations, 6 patients' plain x-rays were positive for soft tissue gas. Here in our study we did plain x-rays only after clinical diagnosis of necrotizing fasciitis. But if we could have done those $\mathrm{x}$-rays as a diagnostic aid in those atypical cases, we could have diagnosed and intervened early in a certain number of patients.

Our study and similar previous studies show most of necrotizing fasciitis patients have high WBC count which is more than 15,000 and high CRP value which is more than 150[3]. As in other previous studies, our study also showed accompanying diabetes in this infection at a higher percentage.

In 9 cases the cause was monobacterial, with a predominance of Pseudomonas aeruginosa in 5 cases, where as in 15 cases cause was polybacterial with a predominance of Streptococcus, staphylococcus and enterobactericae. Similar results were found in other studies also.[7]

With our study we recommend to use basic laboratory investigations including high WBC count, high CRP value, risk factors such as presence of diabetes, advanced age together with presence of soft tissue gas in plain x-rays as a diagnostic aid for atypical presentations of necrotizing fasciitis. And it is possible to develop a cost effective diagnostic score for resource poor settings, which need to be validated in future studies for the early diagnosis of necrotizing fasciitis in difficult cases.

\section{Limitations}

The study was conducted as a retrospective observational study. We included here only the patients who were diagnosed to have necrotizing fasciitis and analysed common findings among them. But to analyse the results in a much more objective way, a control group of people who has similar presentation but not diagnosed to have necrotizing fasciitis is needed. Additionally the sample size was small. Therefore future studies with case control design are necessary to confirm the findings and to formulate a score for early diagnosis of necrotizing fasciitis in atypical presentations.

\section{Conclusion}

A high clinical suspicion of necrotizing fasciitis is needed in an elderly patient presenting with soft tissue infection causing swelling, pain and erythema, with co-morbidity of diabetes. High WBC, High CRP and presence of soft tissue gas in plain $\mathrm{x}$-rays are highly suggestive for underline necrotizing fasciitis. Combining all those findings together will aid in early diagnosis and to formulate a better diagnostic score for the diagnosis of necrotising fasciitis which need to be validated in further studies. Early surgical debridement is the mainstay of treatment.

All authors disclose no conflict of interest. The study was conducted in accordance with the ethical standards of the relevant institutional or national ethics committee and the Helsinki Declaration of 1975, as revised in 2000 .

\section{References}

1. Necrotizing soft tissue infections - UpToDate [Internet]. [cited 2020 Nov 23]. Available from:

https://www.uptodate.com/contents/necrotizing-soft-tissueinfections

2. Tso DK, Singh AK. Necrotizing fasciitis of the lower extremity: Imaging pearls and pitfalls [Internet]. Vol. 91, British Journal of Radiology. British Institute of Radiology; 2018 [cited 2020 Nov 24]. Available from:

/pmc/articles/PMC6209465/?report=abstract

DOI: 10.1259/bjr.20180093

3. Chao WN, Tsai SJ, Tsai CF, Su CH, Chan KS, Lee YT, et al. The Laboratory Risk Indicator for Necrotizing Fasciitis score for discernment of necrotizing fasciitis originated from Vibrio vulnificus infections. J Trauma Acute Care Surg. 2012 Dec;73[6]:1576-82. DOI: 10.1097/TA.0b013e318270d761

4. Necrotizing fasciitis | Radiology Reference Article Radiopaedia.org [Internet]. [cited 2020 Nov 25]. Available from: https://radiopaedia.org/articles/necrotising-fasciitis

5. Goh T, Goh LG, Ang CH, Wong CH. Early diagnosis of necrotizing fasciitis. [cited 2020 Nov 25]; Available from: www.bjs.co.uk DOI: 10.1002/bjs.9371

6. Misiakos EP, Bagias G, Patapis P, Sotiropoulos D, Kanavidis P, Machairas A. Current concepts in the management of necrotizing fasciitis. Front Surg [Internet]. 2014 [cited 2019 Dec 6];1:36. Available from:

http://www.ncbi.nlm.nih.gov/pubmed/25593960

7. Arifi HM, Duci SB, Zatriqi VK, Ahmeti HR, Ismajli VH, Gashi $\mathrm{MM}$, et al. A retrospective study of 22 patients with necrotising fasciitis treated at the University Clinical Center of Kosovo [2005-2010]. Int Wound J [Internet]. 2013 Aug [cited 2019 Dec 6];10[4]:461-5. Available from:

http://www.ncbi.nlm.nih.gov/pubmed/22747529

DOI: 10.1111/j.1742-481X.2012.01005.x

8. Kobayashi T, Morisawa K, Yanai M, Takamatsu Y, Taira Y, Beppu M. 795: RETROSPECTIVE EVALUATION OF NECROTIZING FASCIITIS FOR 5 YEARS. Crit Care Med. 2013;41:A198. DOI: 10.1097/01.ccm.0000440033.83571.4a

9. Leichtle SW, Tung L, Khan M, Inaba K, Demetriades D. The role of radiologic evaluation in necrotizing soft tissue infections. $\mathrm{J}$ Trauma Acute Care Surg. 2016 Nov 1;81 [5]:921-4. DOI: $10.1097 /$ TA.0000000000001244 